\title{
Questioning the Pathogenic Role of the GLA p.Ala143Thr "Mutation" in Fabry Disease: Implications for Screening Studies and ERT
}

\author{
W. Terryn • R. Vanholder • D. Hemelsoet • B.P. Leroy • \\ W. Van Biesen - G. De Schoenmakere • B. Wuyts • \\ K. Claes • J. De Backer • G. De Paepe • A. Fogo • \\ M. Praet • B. Poppe
}

Received: 29 March 2012 /Revised: 30 June 2012 / Accepted: 04 July 2012 / Published online: 29 July 2012

(C) SSIEM and Springer-Verlag Berlin Heidelberg 2013

\begin{tabular}{l}
\hline Communicated by: Ed Wraith \\
\hline Competing interests: none declared \\
\hline W. Terryn ( $\square$ ) \\
Department of Internal Medicine, Division of Nephrology, Regional \\
Hospital Jan Yperman, Ypres, Briekestraat 12, \\
Ieper 8900 , Belgium \\
e-mail:wim.terryn@gmail.com \\
W. Terryn · R. Vanholder • W. Van Biesen \\
Department of Internal Medicine, Division of Nephrology, Ghent \\
University Hospital, Ghent, Belgium \\
D. Hemelsoet \\
Department of Neurology, Ghent University Hospital, Ghent, Belgium \\
B.P. Leroy \\
Department of Ophthalmology, Ghent University Hospital \& Ghent \\
University, Ghent, Belgium \\
G. De Schoenmakere \\
Department of Internal Medicine, Division of Nephrology, Heilig Hart \\
Ziekenhuis, Roeselare, Belgium \\
B. Wuyts \\
Department of Clinical Biology, Laboratory for Metabolic Diseases, \\
Ghent University Hospital, Ghent, Belgium \\
B.P. Leroy • K. Claes · G. De Paepe • B. Poppe \\
Center for Medical Genetics, Ghent University Hospital, Ghent, \\
Belgium \\
J. De Backer \\
Department of Cardiology, Ghent University Hospital, Ghent, \\
Belgium \\
A. Fogo \\
Department of Pathology, Vanderbilt University Medical Center, \\
Nashville, TN, USA \\
M. Praet \\
Department of Pathology, Ghent University Hospital, Ghent, Belgium
\end{tabular}

\begin{abstract}
Fabry disease is an X-linked inborn error of glycosphingolipid metabolism caused by quantitative or qualitative defects in the lysosomal enzyme alfa-Galactosidase A (aGAL A), ultimately resulting in vital organ dysfunction. Mainly the kidneys, the heart, and the central nervous system are involved. While the classical phenotype of Fabry disease is readily recognizable, screening studies have identified clinical variants. Here, we report the phenotype associated with the GLA p.Ala143Thr (c.427G $>$ A) mutation in 12 patients aged 42-83 years. None of the patients had classical Fabry signs or symptoms as angiokeratoma, hypohidrosis, acroparesthesia, or cornea verticillata. Possible Fabry manifestations were renal failure (5/12), stroke (7/12), and left ventricular hypertrophy (5/12), but these were not necessarily attributable to the p.Ala143Thr mutation, as a cardiac biopsy in one female and left ventricular hypertrophy and kidney biopsies in two males with renal failure and microalbuminuria lacked Gb-3 deposits. The literature data on this mutation as well as data collected in the Fabry Outcome Survey (FOS) database confirm these findings. The association of renal failure, stroke, and left ventricular hypertrophy with this mutation could be the result of selection bias, as most patients were detected in screening studies.

We conclude that care should be taken with attribution of vital organ dysfunction to $G L A$ sequence alterations. In case of the p.Ala143Thr mutation, and possibly also other mutations associated with an attenuated phenotype, diagnostic tools such as biopsy and imaging should critically evaluate the relation of end-organ failure with Fabry disease, as this has important consequences for enzyme replacement therapy.
\end{abstract}




\section{Introduction}

Fabry disease (FD, MIM ID \#301500) is an X-linked inborn error of glycosphingolipid metabolism caused by quantitative or qualitative defects in the lysosomal enzyme alfa-Galactosidase A (aGAL A). As a result, glycosphingolipids, mainly globotriaosylceramide (Gb-3), accumulate in different cells throughout the body, ultimately resulting in organ failure (Kint 1970; Brady et al. 1967). Classical FD has been described as a multisystem disease predominantly presenting in males with angiokeratoma, hypohidrosis, and acroparesthesia in childhood, followed by renal failure, left ventricular hypertrophy, stroke, and premature death in the fourth and fifth decade of life. Besides these cases, attenuated forms have been described with a less severe phenotype and a later onset. In males, a specific mutation, associated with a significant residual enzyme activity, can result in a less severe phenotype that presents later in life (e.g., the "cardiac variants") (NAKAO et al. 1995; Scheidt von $W$ et al. 1991). In females, residual enzyme activity can be the consequence of skewed X-chromosome inactivation. In the present paper, we present clinical and pathological data on a series of 12 patients with the p.Ala143Thr mutation and compare these data with literature data (9 patients) and data from the Fabry Outcome Survey (FOS) (20 patients).

\section{Patients and Methods}

We retrospectively reviewed the charts of patients with the p.Ala143Thr mutation diagnosed in our different screening studies and in the subsequent pedigree analyses (Terryn et al. 2008; De Schoenmakere et al. 2008). These studies were conducted according the World Medical Association Declaration of Helsinki Ethical Principles for Medical Research Involving Human and were approved by the Ethics Institution Review Boards of participating centers. All patients gave written informed consent.

Measurement of aGAL A activity was based on a technique involving a dried blood spot sampled on filter paper (DBS) as described by Chamoles et al. (2001). To validate this technique in our laboratory setting, we performed an analysis of 50 control samples (nonnephrology, non-ICU, non-hematology, non-pediatric). In case of low aGAL A activity, DBS was repeated in a new blood sample.

In a second part, previously published cases of the $\mathrm{p}$. Ala143Thr mutation were identified through a PubMed search from 1966 to September 6, 2011, entering "p.Ala143Thr", "A143T," AND "Fabry Disease" as MESH terms.
A third part of this study consists of the analysis of FOS data. FOS - the Fabry Outcome Survey - is a European outcomes database for patients with Fabry disease who are receiving, or are candidates for, Enzyme Replacement Therapy (ERT) with agalsidase alfa. Data from all consenting patients are entered into the database following a structured clinical assessment by a physician or a specialized nurse. FOS has been approved by the Ethics Institution Review Boards of participating centers and all patients gave written informed consent. All measurements performed routinely in clinical practice are entered into the database. Anonymous data are submitted electronically by participating physicians to the central FOS database. We summarized baseline available data on all adult $(18+)$ patients with the p.Ala143Thr mutation in this database.

\section{Kidney Biopsies}

If kidney biopsies were available, they were reviewed by a local pathologist and by a renal pathologist with expertise in FD (AF). Sections were stained with H \& E, PAS, Jones, Congo Red, toluidine blue, and trichrome.

\section{Results}

In total, 41 patients with the $G L A$ p.Ala143Thr mutation were identified.

Twelve patients (three males and nine females) were detected through our screening studies and subsequent pedigree analysis (Table 1). "Classical" symptoms of FD (angiokeratoma, acroparesthesia, cornea verticillata, and hypohydrosis) were absent. A significant number of patients had left ventricular hypertrophy $(N=5)$ or a history of stroke $(N=7)$, but this could be due to a selection bias, as most of these patients were detected as a result of screening studies in populations with left ventricular hypertrophy or stroke (Terryn et al. 2008, accepted in the International Journal of Cardiology). Of note, residual enzyme function could be demonstrated in all patients. Patient 1, 2, and 5 had a kidney biopsy showing no signs of Fabry nephropathy. Proteinuria and renal failure in patient 1 could be attributed to diabetic nephropathy. Patient 2 was detected as a result of pedigree analysis and despite his low aGAL A he had no signs of FD and was asymptomatic besides intermittent paresthesias in both arms, that were aspecific according to an expert neurologist.

One female patient with pronounced left ventricular hypertrophy (LVH) and heart failure (patient 7) had a myocardial biopsy showing AL amyloid and no typical Gb-3 deposition. In our screening studies in high-risk 


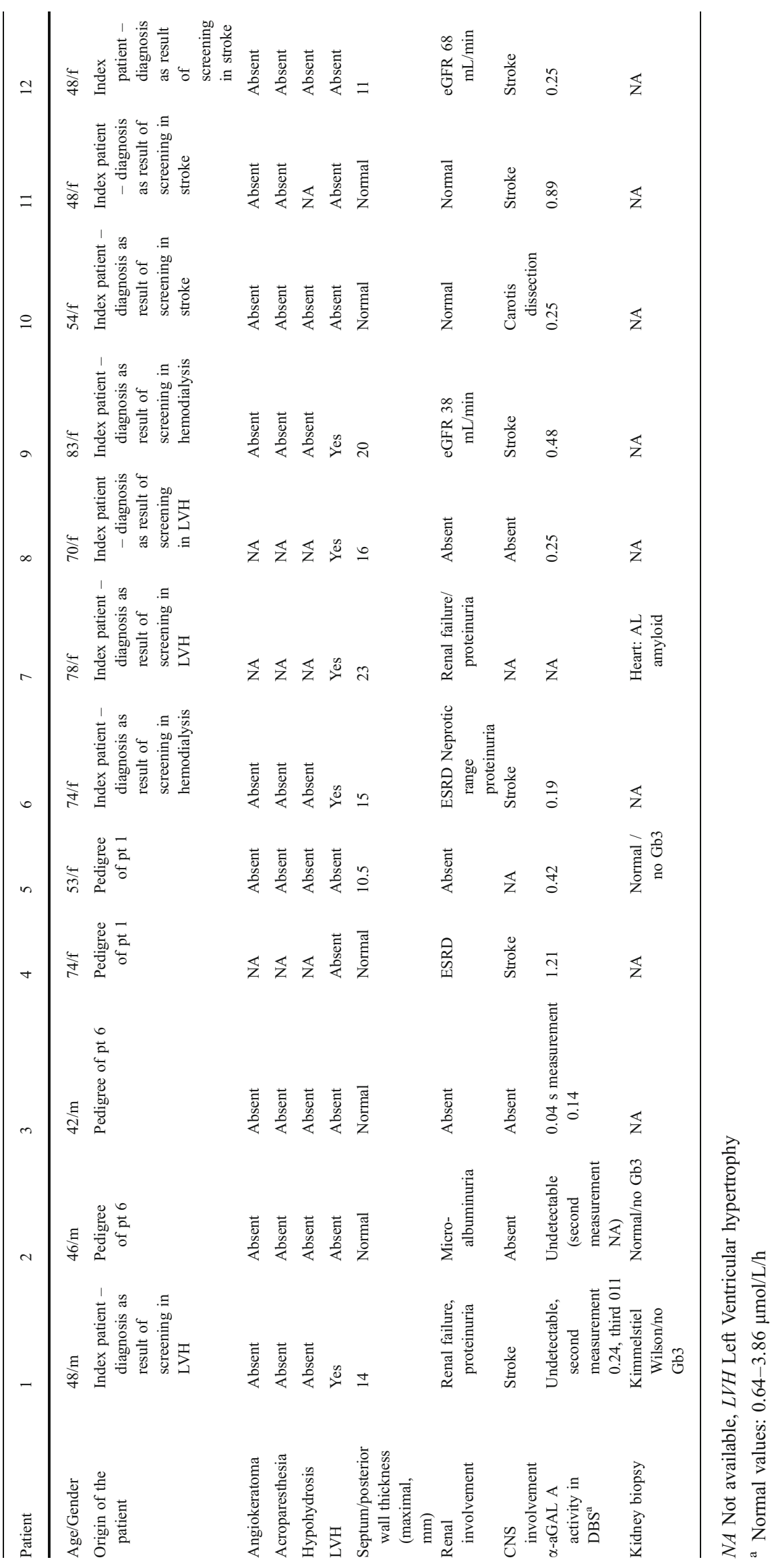


Table 2 Screening in high-risk groups in Flanders

\begin{tabular}{|c|c|c|c|c|}
\hline & Hemodialysis $^{\mathrm{a}}$ & Kidney transplantation ${ }^{\mathrm{b}}$ & Left ventricular hypertrophy $^{c}$ & Total high-risk population \\
\hline Female $(\mathrm{N})$ & 742 & 395 & 178 & 178 \\
\hline Mutation in females $(\mathrm{N})(\%)$ & $2(0.27 \%)$ & 0 & $3(1.7 \%)$ & $5(0.38 \%)$ \\
\hline Male $(\mathrm{N})$ & 180 & 278 & 362 & 820 \\
\hline Mutation in females (N) (\%) & $1(0.56 \%)$ & $1(0.36 \%)$ & $2(0.55 \%)$ & $4(0.49 \%)$ \\
\hline Total patients $(\mathbf{N})$ & 922 & 673 & 540 & 2135 \\
\hline Mutations (N) (\%) & $3(0.33 \%)$ & $1(0.15 \%)$ & $5(0.93 \%)$ & $9(0.42 \%)$ \\
\hline GLA p.Ala143Thr (c. $427 \mathrm{G}>\mathrm{A})$ & 2 & 1 & 4 & $7(0.33 \%)$ \\
\hline GLA p.Trp236Arg $($ c.706T $>C)$ & 1 & 0 & 0 & $1(0.046 \%)$ \\
\hline GLA p.Ala5Glu (c.44C $>$ A) & 0 & 0 & 1 & $1(0.046 \%)$ \\
\hline
\end{tabular}

${ }^{\mathrm{a}}$ Wim Terryn et al. (2008)

${ }^{\mathrm{b}}$ De Schoenmakere et al. (2008)

${ }^{\mathrm{c}}$ Terryn W et al. (2012) Prevalence of Fabry disease in a predominantly hypertensive population with left ventricular hypertrophy. Accepted (June 2012) by the "International Journal of Cardiology"

populations (Table 2), GLA mutations were detected in nine apparently unrelated patients, with seven having the GLA p.Ala143Thr mutation.

The cases with p.Ala143Thr from literature are summarized in Table 3. Only two patients were diagnosed as the result of symptoms and signs (patient 4 and 7). One patient (patient 7) had a single angiokeratome. A second patient (patient 4) had a cramp-fasciculation syndrome. The other seven patients were diagnosed as the consequence of screening studies and had no typical Fabry symptoms. One male (patient 4) had a kidney biopsy. Typical Fabry inclusions were only noted in a few collecting ducts and distal tubules but not in podocytes or in the endothelium. Another male (patient 9) had a nephrectomy after transplantation because of bilateral renal cell carcinoma. Histological examination of the nonmalignant renal tissue showed chronic glomerulonephritis, hyalinization, and severe arteriosclerosis, but no lesions typical for Fabry disease.

The FOS data are summarized in Table 4. Among 1933 registered Fabry patients, 20 adults (12 females and 8 males) from the United Kingdom, Germany, France and Belgium had the p.Ala143Thr mutation. The median baseline eGFR (MDRD) in female patients was $83 \mathrm{~mL} / \mathrm{min} / 1.73 \mathrm{~m}^{2}$ at a mean age of 39 . In males, this was $74 \mathrm{~mL} / \mathrm{min}$ for a median age of 45 .

Only limited data were available on the subsequent evolution of kidney function in these patients before ERT was started. In females, median delta eGFR $(\mathrm{mL} / \mathrm{min} / 1.73$ $\mathrm{m}^{2}$ /year) was -3.3 , which is comparable with literature data on Fabry nephropathy. In males, however, median delta eGFR (mL/min/year) was $+1.35 \mathrm{~mL} / \mathrm{min} / 1.73 \mathrm{~m}^{2} /$ year, which is in contradiction with expected kidney function deterioration in Fabry males which is up to $-12.2 \mathrm{~mL} / \mathrm{min} /$ $1.73 \mathrm{~m}^{2} /$ year (Branton et al. 2002).
Many patients (male and female) had micro-albuminuria. Only three had macro-albuminuria ( $>300 \mathrm{mg} / 24 \mathrm{~h}$ ). The cause of albuminuria was not clear, as only two patients had been biopsied. The male (patient 16) did not show Fabry nephropathy but lupus nephritis, and was successfully treated with immunosuppressive therapy. Stroke was mentioned in only one 80-year-old female (patient 8); at this age, stroke cannot simply be attributed to FD alone.

\section{Discussion}

The p.Ala143Thr mutation is a previously reported missense mutation: resulting from a $G$ to $A$ transition at nucleotide position 247 in exon 3, leading to an Alanine to Threonine substitution and has been reported as being pathogenic (Eng et al. 1997).

The p.Ala143Thr mutation was first reported in 1997 (Eng et al. 1997). The propositus was a 1-month-old male infant serendipitously found to have deficient aGAL A activity with no family history of FD. It was concluded in the same paper that the phenotype associated with this mutation was unknown. In 2002, this mutation was detected in patients as a result of screening in dialysis patients (Spada 2002 JIMD abstract). In a second abstract (Spada 2003), the same author considered this mutation to be related to late-onset end-stage renal disease. From that time, we read in literature this is a "known pathogenic mutation," but it was not supported with biopsy data as proof of its pathogenicity. The association of this mutation with renal failure, as in our screening studies (Table 2) in renal failure or left ventricular hypertrophy, might thus be the result of selection bias.

In vitro expression of this mutant allele in COS 7 cells has been studied (Spada et al. 2006). There is $36 \%$ of 

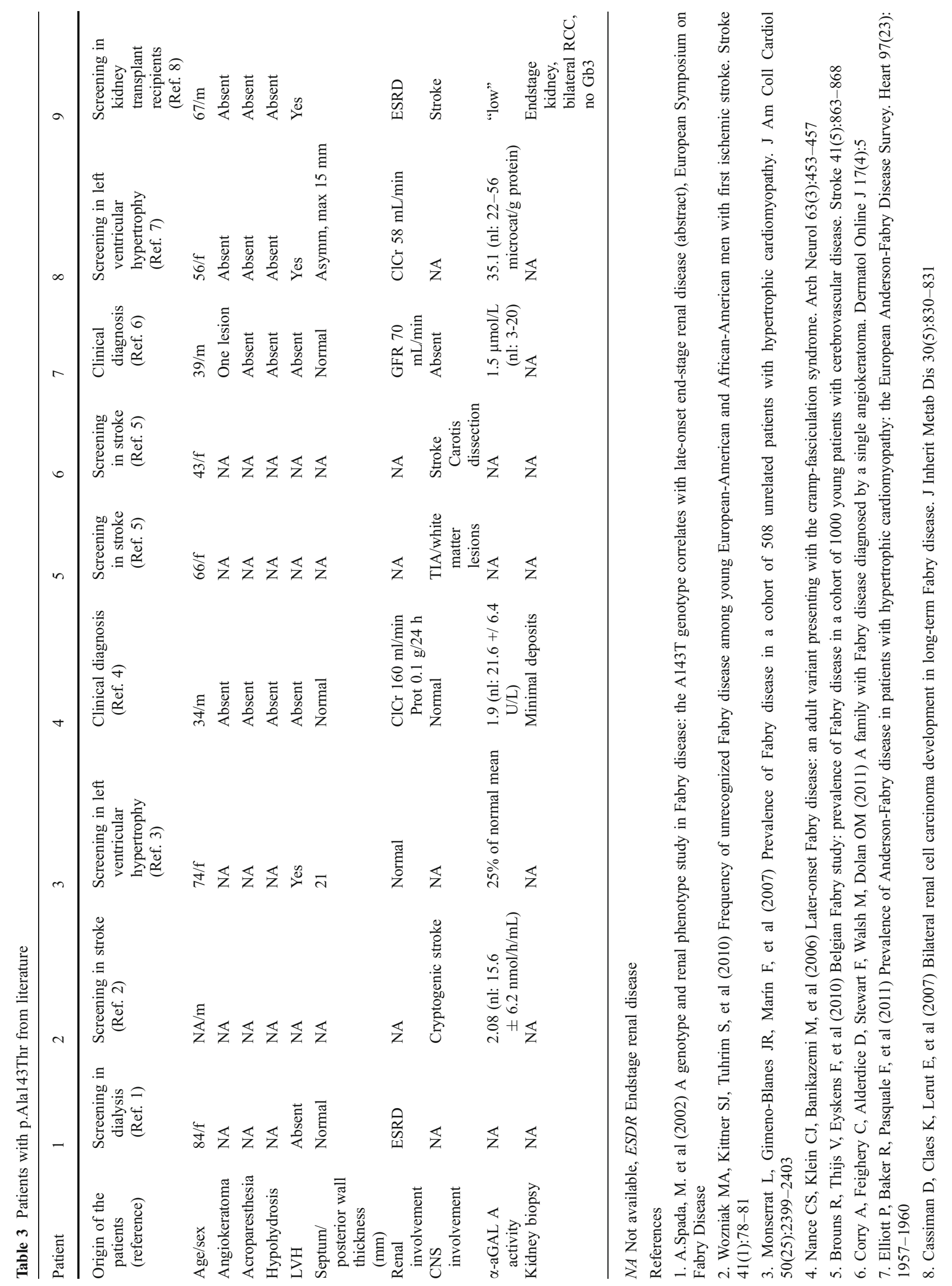
Table 4 FOS data on adult patients with the p.Ala143Thr mutation

\begin{tabular}{|c|c|c|c|c|c|c|c|c|c|c|}
\hline $\begin{array}{l}\text { Patient; } \\
\text { code } \\
\text { FOS }\end{array}$ & Sex & $\begin{array}{l}\text { Age at } \\
\text { baseline }\end{array}$ & $\begin{array}{l}\mathrm{aGAL} A \\
(\mathrm{nmol} / \mathrm{h} / \\
\mathrm{mL})^{\mathrm{a}}\end{array}$ & $\begin{array}{l}\text { eGFR before start } \\
\text { of ERT (MDRD, } \\
\mathrm{mL} / \mathrm{min} / 1.73 \mathrm{~m}^{2} \text { ) }\end{array}$ & $\begin{array}{l}\text { Proteinuria before } \\
\text { start of ERT } \\
(\text { mean })(\mathrm{mg} / 24 \mathrm{~h})\end{array}$ & $\begin{array}{l}\text { Follow up } \\
\text { before ERT } \\
\text { (months) }\end{array}$ & $\begin{array}{l}\text { Delta eGFR } \\
(\mathrm{mL} / \mathrm{min} / \\
1.73 \mathrm{~m} 2 / \\
\text { year) }\end{array}$ & $\begin{array}{l}\text { Kidney } \\
\text { biopsy }\end{array}$ & Stroke & ERT \\
\hline 1 & $\mathrm{~F}$ & 32 & $2.7^{\mathrm{a}}$ & 107 & 165 & 30 & $-3,3$ & No & No & Yes \\
\hline 2 & $\mathrm{~F}$ & 55 & $4.9^{\mathrm{a}}$ & 98 & 230 & 5 & +16.1 & No & No & Yes \\
\hline 3 & $\mathrm{~F}$ & 21 & $0,48^{\mathrm{b}}$ & 108 & 61 & 51 & -6.1 & No & No & Yes \\
\hline 4 & $\mathrm{~F}$ & 29 & $0,22^{\mathrm{b}}$ & 83 & 102 & 10 & +7.0 & No & No & Yes \\
\hline 5 & $\mathrm{~F}$ & 33 & $0,17^{\mathrm{b}}$ & 71 & 107 & 11 & -7.6 & No & No & Yes \\
\hline 6 & $\mathrm{~F}$ & 38 & $0,79^{\mathrm{b}}$ & 82 & 110 & 22 & -1.6 & No & No & No \\
\hline 7 & $\mathrm{~F}$ & 47 & NA & 71 & 1802 & NA & NA & No & No & Yes \\
\hline 8 & $\mathrm{~F}$ & 80 & NA & 27 & NA & 30 & -5.6 & Yes & Yes & No \\
\hline 9 & $\mathrm{~F}$ & 51 & NA & 85 & NA & NA & NA & No & No & No \\
\hline 10 & $\mathrm{~F}$ & 64 & $0,69^{\mathrm{b}}$ & 67 & 140 & 8 & -11.5 & No & No & Yes \\
\hline 11 & $\mathrm{~F}$ & 24 & NA & NA & NA & NA & NA & NA & NA & Yes \\
\hline 12 & $\mathrm{~F}$ & 40 & $12^{\mathrm{a}}$ & 109 & NA & 1 & +69.5 & No & No & No \\
\hline Median & $F$ & 39 & & 83 & & & -3.3 & & & \\
\hline 13 & $\mathrm{M}$ & 26 & $0.5^{\mathrm{c}}$ & 177 & NA & 6 & -2.0 & No & No & Yes \\
\hline 14 & $\mathrm{M}$ & 62 & $0,14^{\mathrm{a}}$ & 67 & 303 & 21 & +5.6 & No & No & Yes \\
\hline 15 & $\mathrm{M}$ & 52 & NA & 74 & 120 & 10 & +4.7 & No & No & Yes \\
\hline 16 & $\mathrm{M}$ & 40 & $0,25^{\mathrm{b}}$ & 15 & 2755 & 2 & +91.3 & Yes & No & NA \\
\hline 17 & $\mathrm{M}$ & 68 & $0,15^{\mathrm{b}}$ & 43 & 185 & 13 & -7.2 & No & No & Yes \\
\hline 18 & $\mathrm{M}$ & 44 & $22^{\mathrm{d}}$ & 112 & 137 & NA & $-8,0$ & No & No & Yes \\
\hline 19 & $\mathrm{M}$ & 46 & $13^{\mathrm{d}}$ & 130 & 128 & NA & NA & No & No & Yes \\
\hline 20 & $\mathrm{M}$ & 45 & 2.4 & NA & $41^{\mathrm{e}}$ & NA & NA & No & No & Yes \\
\hline Median & $M$ & 45 & & 74 & & & +1.35 & & & \\
\hline
\end{tabular}

$N A$ Not available, MDRD Modification of diet in renal disease, ERT Enzyme replacement therapy

${ }^{\mathrm{a}}(\mathrm{nmol} / \mathrm{h} / \mathrm{mL})$ normal values $3.4-13$

${ }^{\mathrm{b}}$ (nU/mg) normal values $0.36-0.84 \mathrm{mU} / \mathrm{mg}$

${ }^{\mathrm{c}}$ Measured shortly at birth

${ }^{\mathrm{d}} \mathrm{nmol} / \mathrm{MU} / \mathrm{mg}$ protein normal $>33$

${ }^{\mathrm{e}} \mathrm{mg} / \mathrm{gr}$ creatinine

expressed aGAL A wild-type activity which is in agreement with residual enzyme function in our patients. The finding of a low aGAL A activity however is not directly related to FD. We found that the p.Ala143Thr mutation indeed is associated with a low aGAL A activity, but its contribution to the phenotype of our patients (stroke, renal failure, left ventricular hypertrophy) is unclear. We performed three kidney biopsies, all lacking typical Gb-3 deposits which are universally present in Fabry patients (Noël et al. 2012). Moreover, among the remaining patients described in this study, we could not find one patient with this mutation and renal failure in whom significant renal Fabry disease was proven by kidney biopsy. In the sphingolipidosis, the ratio of substrate influx into the lysosome and the capacity of the degrading system determines the storage and as such the course and severity of the disease. This is treated in quantitative terms by the so-called threshold theory (Kolter 2011). Only the decrease of enzyme activity below the critical threshold value causes storage of the corresponding lipid substrate. Decrease of enzyme activity to the calculated threshold value does not influence the turnover rate of the substrate (as above this threshold, there is no (linear) relation between enzyme activity and turnover) and pathological storage occurs only below this level. With the exception of acid ceramidase, a decrease of enzyme activity to values of $20 \%$ of normal cells, a typical range for heterozygote carriers of inherited diseases, has no impact on the turnover rate (Kolter 2011).

Our findings corroborate these findings, as we found no deposition of Gb-3 in the lysosomes of the cells of our patients with the p. Ala143Thr mutation. On the basis of the "threshold theory" and the in vitro studies of Spada et al. (2006), 
this could be predicted, as the in vitro expression of aGAL $\mathrm{A}$ in this genotype was $36 \%$ of the wild type expression, which is well over the $20 \%$ mentioned by Kolter 2011.

In FOS, kidney function in patients with p.Ala143Thr remains well preserved in males until their $40 \mathrm{~s}$, which is in contradiction with studies on natural history (Branton et al. 2002). Unfortunately, we have no biopsy data in all of these patients, so we cannot ascertain or exclude renal FD in many patients.

Attenuated Fabry phenotypes lacking the classical FD symptoms have been described as a consequence of residual aGAL A activity. Some mutations result in residual aGAL A activity. This has been described to result in "cardiac variants" that present later in life, with predominantly cardiac manifestations (Scheidt von W et al. 1991). Most of the female patients in this study could be regarded as "variants"; they have significant residual enzyme function, no classical FD symptoms, and mostly cardiac and neurological symptoms.

On the other hand, as FD remains the subject of screening studies in high-risk populations including patients with renal failure and/or stroke, there is a danger of misdiagnosis as a result of selection bias, especially as the p.Ala143Thr mutation was not only detected in screening studies in Belgium (Terryn et al. 2008; De Schoenmakere et al. 2008; Brouns et al. 2010) but also in newborn screenings in Italy (Spada et al. 2006), Taiwan (Lin et al. 2009) Austria (Mechtler et al. 2012), and in other screening studies (Monserrat et al. 2007; Elliott et al. 2011).

The prevalence of the p.Ala143Thr mutation in our highrisk populations $(0.33 \%$, Table 3$)$ is almost 20 times higher than in a European newborn population $(0.017 \%$, Mechtler et al. 2012). Low $\alpha$-aGAL A activity could be one cofactor contributing to endothelial stress, provoking stroke, renal failure, or other signs, and symptoms classically associated with FD. The lack of Gb-3 deposits on electron microscopy does not preclude high intracellullar (lyso)-Gb3 levels that could be pathogenic and cause endothelial cell dysfunction (Namdar et al. 2012), though this should be confirmed with further studies.

Despite the coexistence of renal failure, proteinuria, and low aGAL A activity in patient 1 from our database (Table 1), the diagnosis of Fabry nephropathy was offset by the biopsy that showed a typical case of diabetic nephropathy. Proteinuria and renal failure in patient 16 in the FOS database (Table 4) was secondary to lupus nephritis. The cardiac biopsy in patient 7 from our own database (Table 1) with LVH and heart failure showed AL amyloid. These examples prove that before accepting the diagnosis of FD, confirmation of a mutation and diminished enzyme activity are needed, as well as comprehensive clinical and pathological workup of the patients, where biopsies of the involved organ, next to other diagnostics tools as MRI in left ventricular hypertrophy, should confirm the diagnosis.

Only two patients with the p.Ala143Thr in FOS were reported to have had a kidney biopsy, in spite of a larger number of patients with renal failure. Nevertheless, most of the included patients were treated with ERT. This expensive treatment is possibly not warranted in these patients.

In stroke, establishing a diagnosis of FD is even more difficult as is the case also in several of our own cases, as biopsy of the affected organ is impossible. Diagnosis is especially difficult when other typical features of FD are lacking, as is the case for the p.Ala143Thr mutation.

It has been proposed by expert panels to start treatment in all adult ( $>16$ years) male Fabry patients, and in all patients, pediatric, male, or female, "as soon as clinical signs and symptoms are observed" (Eng et al. 2006). In our cases, however, we have no knowledge on the contribution of the enzymatic defect to the patients' morbidity and no reliable prognostic data are available on the evolution in case of an "atypical" variant like the p.Ala143Thr. It is even questionable if these patients have Fabry disease at all; it has been suggested to call such mutations, biochemically true positive but clinically false positive, as "fringe mutations" (Houge et al. 2011). Moreover, ERT has been studied in the classical phenotype, but there are no studies on the effects in the atypical variants.

The inclusion of patients with the p.Ala143Thr or other mutations associated with an attenuated phenotype (e.g., N215S or p.Asn215Ser, Branton et al. 2002) in studies on the effectiveness of ERT could confound results and should be studied separately. The place of ERT in patients with the p.Ala143Thr mutation is still unclear and should be the subject of close study. The currently available databases such as the industry-sponsored FOS cannot answer this question. An independent international database with mandatory data collection could provide quality data for further study.

Based on our data, we conclude that the expressivity of the p.Ala143Thr mutation is extremely variable. The presence of this mutation is not to be directly associated with pathology, and we have no compelling data that label this mutation as "pathogenic." At most, it is "possibly" pathogenic. As a consequence, biopsy and clinical data should be collected in order to be able to understand the natural evolution and to decide on the need for ERT.

\section{Conflict of Interest}

WT received grants for research from Genzyme Belgium and Shire Belgium. DH received a research grant from Genzyme Belgium. RVH received grants from Genzyme Belgium. BW received funding from Genzyme Belgium. The other authors declare no conflict of interest. 


\section{References}

Brady R, Gal A, Bradley R, Martensson E, Warshan A (1967) Enzymatic defect in Fabry's disease - ceramidetrihexosidase deficiency. N Engl J Med 276:1163-1167, [Internet] Available from: http://www.nejm.org/doi/pdf/10.1056/ NEJM196705252762101

Branton M, Schiffmann R, Kopp JB (2002) Natural history and treatment of renal involvement in Fabry disease. J Am Soc Nephrol 13(Suppl 2):S139-143, [Internet] Available from: http:// eutils.ncbi.nlm.nih.gov/entrez/eutils/elink.fcgi? $\mathrm{db}$ from $=$ pubmed $\& \mathrm{id}=12068026 \&$ retmode $=$ ref $\& \mathrm{cmd}=$ prlinks

Brouns R, Thijs V, Eyskens F et al (2010) Belgian Fabry Study: prevalence of Fabry disease in a cohort of 1000 young patients with cerebrovascular disease. Stroke; J Cerebral Circulation 41(5):863-868, [Internet] Available from: http://stroke. ahajournals.org/cgi/doi/10.1161/STROKEAHA.110.579409

Chamoles NA, Blanco M, Gaggioli D (2001) Fabry disease: enzymatic diagnosis in dried blood spots on filter paper. Clin Chim Acta 308(1-2):195-196

De Schoenmakere G, Poppe B, Wuyts B et al (2008) Two-tier approach for the detection of alpha-galactosidase A deficiency in kidney transplant recipients. Nephrol Dial Transplant 23(12): 4044-4048

Elliott P, Baker R, Pasquale F et al (2011) Prevalence of AndersonFabry disease in patients with hypertrophic cardiomyopathy: the European Anderson-Fabry Disease Survey. Heart 97(23): 1957-1960

Eng CM, Ashley GA, Burgert TS, Enriquez AL, D’Souza M, Desnick RJ (1997) Fabry disease: thirty-five mutations in the alphagalactosidase A gene in patients with classic and variant phenotypes. Mol Med 3(3):174-182

Eng CM, Germain DP, Banikazemi M et al (2006) Fabry disease: guidelines for the evaluation and management of multi-organ system involvement. Genetics Med 8(9):539-548, [Internet] Available from: http://content.wkhealth.com/linkback/openurl? sid $=$ WKPTLP:landingpage $\& a n=00125817-200609000-00001$

Houge G, Tøndel C, Kaarbøe Ø, Hirth A, Bostad L, Svarstad E (2011) Fabry or not Fabry - a question of ascertainment. Eur J Human Genetics 19(11):1111-1112, [Internet] Available from: http:// www.nature.com/doifinder/10.1038/ejhg.2011.87

Kint JA (1970) Fabry's disease: alpha-galactosidase deficiency. Science (New York, NY) 167(3922):1268-1269. [Internet] Available from: http://eutils.ncbi.nlm.nih.gov/entrez/eutils/elink.fcgi? $\mathrm{db}$ from $=$ pubmed $\& \mathrm{id}=5411915 \&$ retmode $=$ ref $\& \mathrm{cmd}=$ prlinks

Kolter T (2011) A view on sphingolipids and disease. Chem Phys Lipids 164(6):590-606
Lin HY, Chong KW, Hsu JH, Yu HC, Shih CC, Huang CH, Lin SJ, Chen $\mathrm{CH}$, Chiang $\mathrm{CC}$, Ho HJ, Lee PC, Kao CH, Cheng $\mathrm{KH}$ Hsueh C, Niu DM (2009) High incidence of the cardiac variant of Fabry disease revealed by newborn screening in the Taiwan Chinese population. Circ Cardiovasc Genet 2(5):450-456

Mechtler TP, Stary S, Metz TF et al (2012) Neonatal screening for lysosomal storage disorders: feasibility and incidence from a nationwide study in Austria. Lancet 379(9813):335-341

Monserrat L, Gimeno-Blanes JR, Marín F et al (2007) Prevalence of Fabry disease in a cohort of 508 unrelated patients with hypertrophic cardiomyopathy. J Amer College Cardiol 50 (25):2399-2403, [Internet] Available from: http://eutils.ncbi. nlm.nih.gov/entrez/eutils/elink.fcgi? dbfrom $=$ pubmed \& $\mathrm{id}=18154965 \&$ retmode $=$ ref $\& \mathrm{cmd}=$ prlinks

Nakao S, Takenaka T, Maeda M et al (1995) An atypical variant of Fabry's disease in men with left ventricular hypertrophy. N Engl J Med 333(5):288-293, [Internet] Available from: http://eutils. ncbi.nlm.nih.gov/entrez/eutils/elink.fcgi?dbfrom $=$ pubmed $\&$ $\mathrm{id}=7596372 \&$ retmode $=$ ref $\& \mathrm{cmd}=$ prlinks

Namdar M, Gebhard C, Studiger R et al (2012) Globotriaosylsphingosine accumulation and not alpha-galactosidase-a deficiency causes endothelial dysfunction in Fabry disease. PLoS ONE 7(4):e36373, [Internet] Available from: http://eutils.ncbi. nlm.nih.gov/entrez/eutils/elink.fcgi? dbfrom $=$ pubmed \& id $=22558451 \&$ retmode $=$ ref $\& \mathrm{cmd}=$ prlinks

Noël L-H, Laurent B, Grünfeld J-P (2012) Renal biopsies in Fabry disease: A multicenter French study. Nephrologie Therapeutique. [Internet] Available from: http://eutils.ncbi.nlm.nih.gov/entrez/ eutils/elink.fcgi?dbfrom $=$ pubmed\&id $=22591703 \&$ retmode $=$ ref $\&$ $\mathrm{cmd}=$ prlinks

von Scheidt W, Eng CM, Fitzmaurice TF et al (1991) An atypical variant of Fabry's disease with manifestations confined to the myocardium. N Engl J Med 324(6):395-399, [Internet] Available from: http://eutils.ncbi.nlm.nih.gov/entrez/eutils/elink.fcgi? dbfrom $=$ pubmed\&id $=1846223 \&$ retmode $=$ ref $\& \mathrm{cmd}=$ prlinks

Spada M, Pagliardini S (2002) Screening for Fabry Disease in endstage nephropathies. J Inherit Metab Dis 25 (Suppl. 1), p 113, Abstract number 226-P

Spada M, Pagliardini S (2003) Prevalence and characteristics of Fabry disease in nephrolgoy: a lesson from the screening of 6378 male patients on dialysis. J Inherit Metab Dis 26 (Suppl 2), p. 145, Abstract number 289-O

Spada M, Pagliardini S, Yasuda M et al (2006) High incidence of later-onset Fabry disease revealed by newborn screening. Am J Hum Genet 79(1):31-40

Terryn W, Poppe B, Wuyts B et al (2008) Two-tier approach for the detection of alpha-galactosidase A deficiency in a predominantly female haemodialysis population. Nephrol Dial Transplant 23(1): 294-300 